\title{
Seguridad en vacunas: descripción de los eventos adversos notificados al sistema de vigilancia en Chile, 2014 a 2016
}

\author{
Oliva Thomsen P. ${ }^{1}$, Adiela Saldaña V. ${ }^{2}$, Jaime Cerda L. ${ }^{3}$ y Katia Abarca V. ${ }^{4}$
}

\author{
Vaccine safety: description of the adverse events reported to the surveillance system \\ in Chile, 2014 to 2016
}

Background: Passive surveillance systems for adverse events following immunization (AEFI) allow the monitoring of known adverse effects (AE) and the investigation of infrequent AE. Aim: To describe the AEFI notified in Chile between 2014 and 2016. Methodology: This was a study of epidemiological surveillance. A general analysis of AEFI and sub-analysis for pentavalent vaccine was carried out. It was calculated the global reporting rate of AEFI every 100,000 doses of vaccines, the reporting rate of AEFI considered of interest and the odds ratio of reporting (ROR) of apneas for pentavalent. Results: The total AEFI reported was 2,552 with a global reporting rate of $9,13.13 .4 \%$ of AEFI were serious. The vaccine reporting rate of events of interest was generally lower than the reference rates. The vaccine with the highest reporting rate (44.3) was pentavalent, with an ROR of apnea versus other vaccines of 10.5 (95\% CI 3.77-29.47). Discussion: This study provides an overview of the AEFI notified in Chile. The global reporting rate was similar to other studies, but those of events of interest were lower than international references. A possible association between apnea and pentavalent vaccine is shown.

Keywords: Pharmacovigilance; passive surveillance; vaccines/adverse effects.

Palabras clave: Farmacovigilancia; vigilancia pasiva; efectos adversos a vacunas.

\section{Introducción}

$\mathrm{L}$ as vacunas son una de las herramientas de mayor impacto en Salud Pública en el mundo, pues han contribuido a disminuir de forma significativa la incidencia de numerosas enfermedades transmisibles'. Gracias a su efectividad, estas enfermedades son cada vez menos frecuentes y van perdiendo importancia en la opinión pública e incluso dentro del equipo de salud. Debido a que las vacunas se administran en población sana, se espera que el beneficio protector supere los efectos adversos (EA) asociados ${ }^{2}$. En consecuencia, cobra gran importancia el evaluar la seguridad de las vacunas mediante la monitorización de los EA asociados a ellas.

Los sistemas de vigilancia en vacunas permiten asegurar el mejor estándar de seguridad en vacunas, buscan monitorizar EA conocidos, pesquisar EA de baja ocurrencia y desconocidos e identificar posibles factores de riesgo para EA específicos. Estos sistemas pueden corresponder a una vigilancia activa o pasiva $^{3}$. En la vigilancia activa se realiza un seguimiento activo y controlado de los eventos que ocurren con posterioridad a la vacunación, por lo que son más difíciles de implementar e implican mayores costos en salud. La vigilancia pasiva se caracteriza por utilizar el reporte espontáneo de los eventos adversos supuestamente atribuidos a vacunas (ESAVI), por lo que es menos costoso, simple y logra abarcar una mayor población y vacunas a monitorizar, siendo el sistema de vigilancia más frecuentemente utilizado en la mayoría de los países, incluyendo a Chile. Los ESAVI corresponden a EA que se manifiestan a continuación de la inmunización y que no pueden ser atribuidos inicialmente a alguna patología específica. Debido a lo anterior, la mayoría de los ESAVI tiene relación cronológica pero no necesariamente causal con la vacunación.

Diversos factores dificultan la interpretación de los datos obtenidos de los sistemas de vigilancia pasiva, como son la sub-notificación, la notificación estimulada (aumento de la notificación relacionados a factores mediáticos), la heterogeneidad en los diagnósticos por parte de los médicos clínicos que notifican y la dificultad de asignación de causalidad a la vacuna como responsable final del EA reportado. Por tales razones, los sistemas de vigilancia pasiva no permiten estimar adecuadamente la incidencia de los EA en la población ${ }^{4,5}$. Entonces, como alternativa a la tasa de incidencia, se utiliza la Tasa de Reporte (TR) de ESAVI, que corresponde al número de ESAVI reportados por cada 100.000 dosis de vacunas administradas. Como medidores de desproporcionalidad para pesquisar señales de asociación entre eventos adversos y la vacuna notificada se utiliza la Razón de Reportes Proporcionales (RRP) o el Odds Ratio de Reporte (ROR) entre otros ${ }^{6,7}$.

En Chile, el sistema de vigilancia en vacunas fue im-
'Departamento de Pediatría, Escuela de Medicina, Pontificia Universidad Católica de Chile. Santiago, Chile.

${ }^{2}$ Subdepartamento de

Farmacovigilancia, Instituto de Salud Pública. Santiago, Chile. ${ }^{3}$ División de Salud Pública y Medicina Familiar, Escuela de Medicina, Pontificia Universidad Católica de Chile. Santiago, Chile. ${ }^{4}$ Departamento de Enfermedades Infecciosas e Inmunología Pediátricas, Escuela de Medicina, Pontificia Universidad Católica de Chile. Santiago, Chile.

Los establecimientos en que se llevó a cabo este proyecto de investigación fueron: - Pontificia Universidad Católica de Chile, Santiago, Región Metropolitana, Chile. - Instituto de Salud Pública de Chile, Nuñ̃oa, Región Metropolitana, Chile.

Los autores no presentan conflictos de interés.

Este proyecto no recibió financiamiento.

Recibido: 20 de marzo de 2018 Aceptado: 9 de abril de 2019

Correspondencia a: Katia Abarca Villaseca katia@med.puc.cl 
plementado en 1995 y actualmente se encuentra a cargo del Sub-Departamento de Farmacovigilancia (SDFV) del Instituto de Salud Pública (ISP) y del Departamento de Inmunizaciones del MINSAL. La notificación se realiza en un formulario de notificación conjunta ESAVI/EPRO (errores programáticos), el cual se puede realizar en forma escrita o vía electrónica. La notificación es obligatoria para los profesionales relacionados con la vacunación ${ }^{8}$.

Dentro de las vacunas incluidas en el Programa Nacional de Inmunizaciones (PNI) de Chile, a la fecha del estudio se encontraba la vacuna pentavalente (toxoides diftérico y tetánico, vacuna pertussis de células completas, antígeno de superficie hepatitis B y vacuna conjugada de Haemophilus influenzae tipo b) la que tiene una de las mayores TR comparada con otras vacunas, específicamente debida al componente pertussis ${ }^{4}$. En la literatura científica se ha descrito que esta vacuna podría causar apnea, aunque aún no se ha establecido una relación causal definitiva ${ }^{9}$. Por otro lado, se ha observado que en los pacientes prematuros este riesgo parece exacerbado ${ }^{10}$.

El objetivo primario de este estudio fue determinar la TR de ESAVI en Chile entre los años 2014 y 2016. Fueron objetivos secundarios describir los tipos de ESAVI más frecuentes, su distribución por grupo etario y los profesionales que más notifican; calcular TR para cada vacuna del PNI, TR según género y región, TR para ESAVI serios; describir los reportes de muertes; comparar las TR de interés nacional con las descritas internacionalmente; y realizar un sub-análisis para la vacuna pentavalente.

\section{Material y Método}

Este proyecto corresponde a un estudio de vigilancia epidemiológica realizado en base a los datos del Sistema de Vigilancia en Vacunas de Chile que recopila el SDFV del ISP.

Previo al análisis de los datos se revisó la base de datos, eliminando las notificaciones duplicadas y se corrigieron inconsistencias. Se excluyó el análisis de las vacunas no incluidas en el PNI debido a que, tanto la notificación de los ESAVI como el registro de dosis de vacunas administradas en el Registro Nacional de Inmunizaciones (RNI), son menos sistemáticos en nuestro medio. Por su parte, no se pudo realizar un análisis por separado de la vacuna neumocócica conjugada y polisacárida ya que son ingresadas indiferenciadamente en la base de datos, ni entre vacuna VPH bivalente y tetravalente, debido a que se notifican en la mayoría de los casos como vacuna VPH sin señalar si es bivalente o tetravalente. En el caso de notificaciones para múltiples vacunas se consideraron todas ellas como posibles causantes del ESAVI, asignando el mismo evento a cada una de ellas.

Para la descripción general de los ESAVI se consideró el período 2014-2016. Existía previo a este proyecto un aumento del número absoluto de ESAVI para la vacuna pentavalente entre los años 2014 (269 ESAVI totales) y 2016 (559 ESAVI totales) observado en el SDFV, en concomitancia con cambios en proveedor de esta vacuna. En contexto de lo anterior, se realizó el análisis de la vacuna pentavalente en dos períodos de 17 meses: período-1 entre enero 2014 y mayo 2015, y período-2 entre enero 2016 y mayo 2017.

\section{Definiciones}

La gravedad de los ESAVI fue clasificada en eventos menores y eventos graves según la definición entregada por el SDFV del ISP, que se basa en la definición propuesta por la asociación International Conference on Harmonization $(\mathrm{ICH})^{8}$. Los eventos menores corresponden a una reacción esperable, leve y auto-limitada. Los eventos graves se definen como aquellos que producen riesgo vital o muerte, hospitalización o prolongación de ésta, secuelas o discapacidades persistentes que afectan la funcionalidad del individuo, anomalías congénitas o defectos de nacimientos, o bien requieren tratamiento para prevenir daño persistente o discapacidad.

Para los reportes de muertes se describieron los diagnósticos señalados por el notificador y el análisis de causalidad realizado por el comité de farmacovigilancia del ISP, que evalúa la plausibilidad biológica de la asociación causal de la vacuna con el evento y la existencia de causas alternativas que expliquen el evento.

Los ESAVI de interés fueron elegidos en base a los EA descritos por la $\mathrm{OMS}^{11}$ para las vacunas incluidas en el PNI de Chile. La definición de caso de estos ESAVI se basó en las recomendaciones de la Brigthon Collaboration $^{12}$. Para la búsqueda y selección de los ESAVI de interés se utilizó el diccionario WHO-ART, se eligieron los términos incluidos más cercanos a la definición del ESAVI de interés y se realizó una búsqueda en la base de datos. Se analizó la descripción realizada en cada notificación, y se excluyeron aquellos eventos que no coincidían con las recomendaciones de la Brigthon Collaboration.

\section{Análisis estadístico}

Se calculó la TR por año, región por vacuna incluida en el PNI y para cada ESAVI de interés. Para estimar el número de dosis administradas por vacunas, se utilizó la información otorgada por el RNI. Para el análisis por grupo etario se evaluó el número y porcentaje de ESAVI; sin embargo, no se calculó la TR ya que no se contaba con las dosis de vacunas administradas a cada grupo.

Para estimar una posible asociación entre la vacuna pentavalente y el evento de apneas ${ }^{7}$, se utilizó el ROR, ya que podría disminuir el riesgo de sesgo en comparación a los otras medidas disponibles ${ }^{13}$. Éste se calculó utilizando el método estadístico para la detección de señales 
en sistemas de reporte espontáneo ${ }^{5,14}$ y corresponde a la razón entre el odds de que ocurra una apnea en pacientes vacunados con pentavalente y el odds de que ocurra una apnea en pacientes que recibieron otras vacunas. No es equivalente a un odds ratio calculado en un estudio controlado por lo que no asegura una asociación, pero permite sugerir una potencial señal que debe ser confirmada en otros estudios.

\section{Consideraciones éticas}

El estudio fue aprobado por el Comité Ético Científico de la Facultad de Medicina de la Pontificia Universidad Católica de Chile. Se entregó una dispensa de consentimiento informado ya que la información utilizada se encuentra anonimizada. Además, se realizó una declaración de responsabilidad y compromiso de confidencialidad de los autores ante el ISP para la entrega de datos de vigilancia destinadas a estudios e investigación científica.

\section{Resultados}

\section{Descripción general de los ESAVI}

El número total de ESAVI notificados entre 2014 y 2016 fue de 2.552 , con una TR de 9,13 por 100.000 dosis de vacunas administradas. El número absoluto de reportes anuales aumentó a través de los años; no obstante, la TR se mantuvo estable en 8,1 entre los años 2014 y 2015, y aumentó a 11,2 el 2016 (Tabla 1). Los ESAVI más notificados fueron eritema, dolor, fiebre e hinchazón. El grupo etario con más notificaciones fue el de los lactantes bajo un año de edad (Tabla 2). Las notificaciones correspondían en $58,7 \%$ a mujeres, $39,7 \%$ a varones y en $1,6 \%$ no fue registrado el género. Las notificaciones fueron realizadas principalmente por enfermeras/os $(70,4 \%)$, seguidos por médicos $(15,5 \%)$ y empresas farmacéuticas $(4,1 \%)$. La Región Metropolitana presentó el mayor número de notificaciones; sin embargo, la mayor TR correspondió a la Región de Aysén (Tabla 3).

Del total de ESAVI, 336 (13,3\%) correspondían a casos clasificados como graves por el responsable de la notificación, con una TR de 1,22 ESAVI serios por 100.000 dosis administradas. Las causas de gravedad de los ESAVI fueron: causa o prolonga hospitalización $(60,42 \%)$, no descrito $(32,15 \%)$, provoca incapacidad $(3,57 \%)$, amenaza la vida $(2,08 \%)$, otra condición médica importante $(1,94 \%)$ y muerte $(0,60 \%)$.

En este período de tres años se notificaron dos muertes, una notificada para vacuna pentavalente y otra para la vacuna neumocócica conjugada (Synflorix ${ }^{\circledR}$ ). El primer caso, un lactante de 2 meses de edad que había recibido la primera dosis de vacuna pentavalente una semana antes de enfermar, tuvo diagnóstico final de coqueluche grave, hipertensión pulmonar refractaria, shock cardiogénico y
Tabla 1. Número y tasa de reporte de ESAVI, según el sistema de vigilancia pasiva del ISP (Chile, 2014-2016)

\begin{tabular}{ccccc}
\hline Año & $\begin{array}{c}\mathbf{n} \text { total de } \\
\text { ESAVI }\end{array}$ & $\begin{array}{c}\text { TR anual } \\
(\mathbf{1 : 1 0 0 . 0 0 0 )}\end{array}$ & $\begin{array}{c}\mathbf{n} \text { total de ESAVI } \\
\text { graves }\end{array}$ & $\begin{array}{c}\text { TR anual de ESAVI } \\
\text { graves }(\mathbf{1 : 1 0 0 . 0 0 0})\end{array}$ \\
\hline 2014 & 672 & 8,13 & 74 & 0,90 \\
2015 & 845 & 8,11 & 124 & 1,19 \\
2016 & 1.005 & 11,22 & 138 & 1,54 \\
Total & 2.533 & 9,13 & 336 & 1,22 \\
\hline
\end{tabular}

ESAVI: efecto supuestamente atribuido a vacunación e inmunización. ISP: Instituto de Salud Pública. TR: tasa de reporte.

Tabla 2. Distribución de ESAVI por grupos etarios, según sistema de vigilancia pasiva del ISP (Chile, 2014-2016)

\begin{tabular}{lccc}
\hline Edad & \% ESAVI (n) 2014 & \% ESAVI (n) 2015 & \% ESAVI (n) 2016 \\
$<1$ año & $38,8 \%(261)$ & $42,3 \%(357)$ & $42,3 \%(425)$ \\
1 - 6 años & $20,5 \%(138)$ & $25,0 \%(211)$ & $18,9 \%(190)$ \\
7 - 17 años & $4,5 \%(30)$ & $7,1 \%(60)$ & $9,8 \%(98)$ \\
$18-64$ años & $29,6 \%(199)$ & $19,1 \%(161)$ & $13,4 \%(135)$ \\
$>$ 64 años & $4,0 \%(27)$ & $5,3 \%(45)$ & $5,3 \%(53)$ \\
No señala & $2,5 \%(17)$ & $1,3 \%(11)$ & $10,4 \%(104)$ \\
Total & $100 \%(672)$ & $100 \%(845)$ & $100 \%(1.005)$
\end{tabular}

ESAVI: efecto supuestamente atribuido a vacunación e inmunización. ISP: Instituto de Salud Pública.

Tabla 3. Número y tasas de reporte anual de ESAVI por regiones, según sistema de vigilancia pasiva del ISP (Chile, 2014-2016)

\begin{tabular}{lcc}
\hline Regiones de Chile & n ESAVI & TR (1:100.000) \\
Región Arica y Parinacota & 12 & 3,00 \\
Región de Tarapacá & 49 & 7,02 \\
Región de Antofagasta & 51 & 5,57 \\
Región de Atacama & 40 & 6,00 \\
\hline Región de Coquimbo & 40 & 3,24 \\
Región de Valparaíso & 343 & 12,70 \\
\hline Región Metropolitana & 1.117 & 10,64 \\
Región del Libertador B. O'Higgins & 48 & 3,26 \\
\hline Región del Maule & 82 & 4,63 \\
\hline Región del Biobío & 242 & 7,58 \\
Región de la Araucanía & 200 & 12,20 \\
Región de los Ríos & 44 & 7,17 \\
Región de los Lagos & 136 & 9,84 \\
\hline Región de Aysén del General Carlos Ibáñez del Campo & 81 & 44,73 \\
\hline Región de Magallanes & 48 & 17,81 \\
\hline ESAVI: efecto supuestamente atribuido a vacunación e inmunización. ISP: Instituto de Salud \\
\hline Pública. TR: tasa de reporte. & & \\
\hline
\end{tabular}


Tabla 4. Número y tasas de reporte de ESAVI y dosis de vacunas de las vacunas incluidas en el PNI, según sistema de vigilancia pasiva del ISP (Chile, 2014-2016)

\begin{tabular}{|c|c|c|c|}
\hline Vacunas PNI & n total de ESAVI & $\begin{array}{l}\text { Dosis total de } \\
\text { vacuna }\end{array}$ & TR $(1: 100.000)$ \\
\hline Pentavalente & 1.199 & 2.706 .488 & 44,30 \\
\hline Toxoides diftérico y tetánico & 26 & 100.729 & 25,81 \\
\hline Polio oral & 495 & 2.079 .556 & 23,80 \\
\hline Neumocócica & 583 & 2.731 .798 & 21,34 \\
\hline VPH & 137 & 875.106 & 15,66 \\
\hline BCG & 96 & 703.881 & 13,64 \\
\hline Meningocócica conjugada & 76 & 796.010 & 9,55 \\
\hline Tresvírica & 160 & 2.399 .241 & 6,67 \\
\hline Tdap & 85 & 1.326 .990 & 6,41 \\
\hline Hepatitis A & 10 & 189.755 & 5,27 \\
\hline Rábica & 35 & 727.940 & 4,81 \\
\hline Influenza estacional & 486 & 11.699 .870 & 4,15 \\
\hline \multicolumn{4}{|c|}{$\begin{array}{l}\text { ESAVI: efecto supuestamente atribuido a vacunación e inmunización. PNI: Programa Nacional } \\
\text { de Inmunizaciones ISP: Instituto de Salud Pública. VPH: virus papiloma humano. BCG: vacuna } \\
\text { Bacillus Calmette-Guerin. Tdap: toxoide diftérico y tetánico y pertussis acelular de contenido } \\
\text { antigénico reducido. TR: tasa de reporte. }\end{array}$} \\
\hline
\end{tabular}

síndrome compartimental abdominal. La vacuna pentavalente es una vacuna inactivada, siendo implausible que hubiese generado esta enfermedad; por otro lado, una buena protección para la enfermedad se logra con al menos tres dosis de vacuna ${ }^{15,16}$. El segundo caso, paciente con 1 año 10 meses de edad, con esquema de tres dosis de vacuna 10 valente, que fue notificado con diagnóstico final de meningitis neumocócica con identificación de Streptococcus pneumoniae serotipo $24 \mathrm{~F}$ en el LCR. La vacuna recibida también es inactivada y no contiene el serotipo causante de la enfermedad de este niño. Por tanto, en ambos casos no hay plausibilidad biológica para asignar una causalidad a la vacuna.

La vacuna con mayor número de notificaciones de ESAVI y mayor TR fue la pentavalente, seguida por toxoide tetánico-diftérico, anti-poliomielítica oral y neumocócica (Tabla 4). Todas las TR de los ESAVI de interés fueron menores que las tasas reportadas internacionalmente ${ }^{17}$, a excepción de la poliomielitis paralítica relacionada a vacuna cuya TR está dentro de los rangos descritos (Tabla 5). Dentro de los ESAVI de interés no existieron notificaciones para osteítis por vacuna $\mathrm{BCG}$, anafilaxia o

Tabla 5. Número y tasa de reporte de ESAVI de interés según sistema de vigilancia pasiva del ISP (Chile, 2014-2016) y sus respectivas tasas de reporte internacional

\begin{tabular}{|c|c|c|c|c|c|}
\hline Vacuna & ESAVI de interés & $\begin{array}{c}\text { Número total de } \\
\text { ESAVI }\end{array}$ & $\begin{array}{l}\text { Dosis total de } \\
\text { vacuna }\end{array}$ & $\operatorname{TR}(1: 100.000)$ & $\begin{array}{l}\text { TR referencia } \\
(1: 100.000)\end{array}$ \\
\hline \multirow[t]{3}{*}{ BCG } & Linfadenitis supurativa & 15 & 703.881 & 2,13 & $10-100$ \\
\hline & Osteítis por BCG & 0 & 703.881 & 0,00 & $1-700$ \\
\hline & BCG diseminada & 1 & 703.881 & 0,14 & $1,56-4,29$ \\
\hline \multirow[t]{4}{*}{ Toxoides diftérico y tetánico } & Anafilaxia & 0 & 923.021 & 0,00 & 0,16 \\
\hline & Absceso estéril & 4 & 923.021 & 0,43 & $0,6-1,0$ \\
\hline & Neuritis braquial & 0 & 923.021 & 0,00 & 0,0069 \\
\hline & Celulitis & 30 & 923.021 & 3,25 & Sin información \\
\hline \multirow[t]{2}{*}{ Hepatitis B } & Anafilaxia & 0 & 217.200 & 0,00 & $0,11-0,20$ \\
\hline & Síndrome de Guillain Barré & 0 & 217.200 & 0,00 & 0,50 \\
\hline Polio oral & Poliomelitis paralítica relacionada a vacuna & 3 & 2.079 .556 & 0,14 & $0,029-0,14$ \\
\hline \multirow[t]{4}{*}{ Tresvírica } & Anafilaxia & 2 & 2.399 .241 & 0,08 & 0,35 \\
\hline & Encefalopatía & 0 & 2.399 .241 & 0,00 & 0,10 \\
\hline & Convulsión febril & 12 & 2.399 .241 & 0,50 & $34,31-86,95$ \\
\hline & Trombocitopenia & 1 & 2.399 .241 & 0,04 & $2,50-3,33$ \\
\hline Influenza estacional & Síndrome de Guillain Barré & 5 & 11699870 & 0,04 & $0,10-0,20$ \\
\hline \multirow[t]{3}{*}{ Neumocócica } & Convulsión febril & 66 & 11.731 .798 & 0,56 & $10-100$ \\
\hline & Encefalopatía & 0 & 11.731 .798 & 0,00 & $10-100$ \\
\hline & Apnea & 25 & 11.731 .798 & 0,21 & $100-1.000$ \\
\hline \multirow[t]{2}{*}{ VPH } & Artralgias & 7 & 875.106 & 0,80 & $<500$ \\
\hline & Síncope & 24 & 875.106 & 2,74 & 8,20 \\
\hline
\end{tabular}

ESAVI: efecto supuestamente atribuido a vacunación e inmunización. ISP: Instituto de Salud Pública. BCG: vacuna Bacillus Calmette-Guerin. VPH: virus papiloma humano. TR: tasa de reporte 
neuritis braquial por vacuna antitetánica/diftérica, anafilaxia o Síndrome de Guillain Barré por vacuna hepatitis $\mathrm{B}$, encefalitis por vacuna tresvírica ni encefalopatía por vacuna neumocócica.

\section{Vacuna pentavalente}

La TR de EA relacionados a la vacuna pentavalente del período 1 (enero de 2014 a mayo de 2015) fue de 28,81 , aumentando a 55,46 en el período 2 (enero de 2016 a mayo de 2017). A pesar que también aumentó la TR de eventos serios de 5,58 en el período 1 a 10,27 en el período 2, éstos representaban el mismo porcentaje con respecto al total de eventos notificados.

Al comparar las TR de ESAVI de interés, se observó un aumento en el período 2 de llanto inconsolable, convulsiones, celulitis, anafilaxia y apneas en prematuros. A pesar de lo anterior, todas las TR en Chile son menores que las descritas internacionalmente (Tabla 6).

Se encontró un total 37 casos de apneas asociadas a vacuna pentavalente, siendo 14 eventos del período 1 y 23 del período 2. El ROR de presentar una apnea en las notificaciones realizadas para la vacuna pentavalente fue de 12,63 (IC 95\% 2,85 - 55,89) en el período 1 y de 9,66 (IC 95\% 2,27 - 41,14) en el período 2; con un ROR global de 10,54 (IC 95\% 3,77 - 29,47) para ambos períodos, comparado con las notificaciones realizadas para otras vacunas (Tabla 7).

\section{Discusión}

Este estudio entrega una visión general de los reportes de ESAVI en Chile del período 2014 a 2016. Es importante recordar que la información debe ser interpretada con precaución, ya que estos datos describen eventos supuestamente atribuidos a la vacunación, pero no indican causalidad. El valor obtenido de TR en Chile (9,1 ESAVI por cada 100.000 dosis administradas), así como el porcentaje de ESAVI serios $(13,4 \%)$ son muy similares a lo reportado por el sistema de vigilancia usado en E.U.A. (VAERS) (TR 11,4 ESAVI por 100.000 dosis administradas y $14,2 \%$ ESAVI serios) ${ }^{4}$.

La edad de mayor notificación de ESAVI fue bajo 1 año de edad, lo que es esperable debido a que corresponde a la edad con mayor número de dosis de vacunas, y a la mayor frecuencia de fiebre y otros EA reportada a esta edad ${ }^{18}$. La región con mayor tasa de notificación fue la Región de Aysén; ya que este estudio obtiene sus datos de un sistema de vigilancia pasiva, la mayor tasa de notificación podría deberse a una mayor adherencia a la notificación, siendo menos probable un mayor riesgo de EA en dicha región. Se requieren otros estudios para saber esto con certeza.

Las TR de ESAVI de interés en Chile fueron menores que las observadas internacionalmente. En general, las
Tabla 6. Tasa de reporte de ESAVI de interés de la vacuna pentavalente según sistema de vigilancia pasiva del ISP en dos períodos en Chile

\begin{tabular}{|c|c|c|c|}
\hline ESAVI de interés & $\begin{array}{c}\text { TR enero } 2014 \\
\text { a mayo } 2015 \\
(1: 100.000)\end{array}$ & $\begin{array}{c}\text { TR enero } 2016 \\
\text { a mayo } 2017 \\
(1: 100.000)\end{array}$ & $\begin{array}{l}\text { TR referencia } \\
(1: 100.000)\end{array}$ \\
\hline Anafilaxia & 0,08 & 0,24 & 0,13 \\
\hline Encefalopatía & 0,08 & 0,08 & $0,018-0,320$ \\
\hline Síndrome hipotonía hiporespuesta & 0,93 & 0,80 & $0-291$ \\
\hline Llanto inconsolable & 7,59 & 10,03 & 3.500 \\
\hline Convulsiones & 4,26 & 6,26 & $8-60$ \\
\hline Apneas & 1,01 & 1,85 & Sin información \\
\hline Apneas en prematuros & 0,15 & 1,12 & $0-47.000$ \\
\hline Celulitis & 1,01 & 4,90 & Sin información \\
\hline
\end{tabular}

ESAVI: efecto supuestamente atribuido a vacunación e inmunización. ISP: Instituto de Salud Pública.

Tabla 7. Odds ratio de reporte (ROR) de apneas para la vacuna pentavalente según sistema de vigilancia pasiva del ISP en dos períodos en Chile

\begin{tabular}{lccccc|}
\hline \multicolumn{5}{c}{ Período 1 - Enero 2014 a mayo 2015 } \\
Vacuna & Apneas & Otros ESAVI & Total & ROR & IC 95\% \\
Pentavalente & 14 & 358 & 372 & 12,63 & $2,85-55,89$ \\
Otras vacunas & 2 & 646 & 648 & & \\
Total & 16 & 1.004 & 1.020 & \\
\multicolumn{5}{c}{ Período 2 - Enero 2016 a mayo 2017 } \\
Vacuna & Apneas & Otros ESAVI & Total & ROR & IC 95\% \\
Pentavalente & 23 & 668 & 691 & 9,66 & $2,27-41,14$ \\
Otras vacunas & 2 & 561 & 563 & & \\
Total & 25 & 1.229 & 1.254 & \\
\multicolumn{5}{c}{} \\
\hline ESAVI: efecto supuestamente atribuido a vacunación e inmunización. ISP: Instituto de Salud \\
Pública.
\end{tabular}

tasas de referencia propuestas por la OMS están extraídas de estudios controlados ${ }^{11}$, lo que explica el menor valor observado en los sistemas de vigilancia pasiva. La utilidad de comparar las TR locales con los valores de referencia es generar señales de alerta en el caso de ser mayores. En este estudio no se identificó ninguna TR mayor a la referencia. Sin embargo, las menores TR también pueden deberse a sub-notificación o a que el número de dosis de vacunas administradas sea menor que el necesario para lograr observar eventos de muy rara ocurrencia ${ }^{3}$.

A diferencia de la mayoría de los ESAVI de interés, la TR de referencia para poliomielitis paralítica relacionada a vacuna es extraída de estudios basados en sistemas de vigilancia pasiva ya que su incidencia tan baja dificulta su pesquisa en estudios controlados que cuentan con un menor número de pacientes ${ }^{19,20}$. Considerando lo anterior, 
el hecho que la TR de poliomielitis paralítica relacionada a vacuna en Chile esté dentro de los rangos observados en el extranjero, sugiere que la sensibilidad de nuestro sistema para pesquisar este EA es comparable con los sistemas internacionales. Probablemente contribuye a ello la vigilancia de parálisis fláccida y la relevancia clínica de esta entidad.

En los casos de notificación de muerte asociada a la vacuna pentavalente y neumocócica conjugada, el análisis de causalidad fue inconsistente por no existir plausibilidad biológica; de hecho ambas notificaciones corresponden más bien a falta de protección por la vacuna (en el primer caso por estar expuesto a Bordetella pertussis antes de completar el esquema de vacunación y en el segundo por carecer la vacuna de cobertura al serotipo causante de la enfermedad) y no a muertes ocurridas a causa de ésta.

El aumento observado en la TR de EA relacionados a la vacuna pentavalente pudiese estar asociado a un cambio del fabricante que abasteció la vacuna en el segundo período. A pesar de que no existía certeza de esta asociación, como medida precautoria el Departamento de Inmunizaciones del MINSAL decidió volver a utilizar el producto del fabricante anterior. Eso ejemplifica que ciertas señales obtenidas por los sistemas de vigilancia pueden servir de base para toma de decisiones por parte de las autoridades sanitarias.

Las apneas son un evento adverso conocido posterior a la inmunización de prematuros ${ }^{21,22}$; dentro de las posibles causantes se ha estudiado específicamente la asociación con las vacunas que poseen componente pertussis ${ }^{10}$. En este estudio se observó una posible asociación de apnea en relación a la vacuna pentavalente, tanto en pacientes de término como prematuros.

Las limitaciones de este estudio son las inherentes a los sistemas pasivos de farmacovigilancia en vacunas. Se reconoce que estos sistemas están sujetos a una alta tasa de sub-notificación, situación que también sugieren nuestros resultados. Se pudo comprobar asimismo frecuentes fallas de registro que entorpecen la interpretación de la información y que requieren de una revisión de los datos antes de ser analizados para intentar disminuir el sesgo de información. Por otra parte, se constató un problema de definición de casos de los eventos reportados, los que son, en general, inespecíficos e incompletos, lo que no permite su rápido análisis. Además, a pesar de existir múltiples efectos ya conocidos y descritos internacionalmente, muchas de las notificaciones no son realizadas con el diagnóstico preciso.

A pesar de sus limitaciones, los sistemas de vigilancia son perfectibles. Es por ejemplo altamente deseable una mayor capacitación del personal de salud respecto a la importancia de notificar los ESAVI y de aportar información completa, así como mejorar el conocimiento de las definiciones operativas del sistema (definición de caso de
EA, criterios de gravedad, etc.). Los propios formularios de notificación pueden ser mejorados. Por ejemplo, la base de datos nacional de ESAVI en Chile incluye las 25 variables sugeridas por la OMS para los sistemas de monitoreo de ESAVI, las que no contemplaban la prematuridad $^{23}$. Considerando que la falta de este antecedente limitaba el análisis de apneas en el sistema nacional, en el año 2017 el SDFV del ISP decidió agregarlo como un ítem obligatorio en la base de datos de ESAVI y en el seguimiento de casos graves, lo que denota una mejora en base la información local.

Una de las fortalezas del sistema chileno es que el denominador utilizado para el cálculo de las TR es bastante confiable, gracias al sistema implementado en los últimos años por el RNI. Aun así, debido a que no corresponde a un seguimiento controlado de esos pacientes, no es completamente certero. Por todo lo anterior, esta información, como cualquier otro sistema de vigilancia pasiva, está sujeta a un alto riesgo de sesgo y no permite el cálculo de incidencia, riesgo relativo o aplicar test de significancia estadística; no obstante, la vigilancia pasiva posee la ventaja de poder levantar potenciales señales como las descritas en este trabajo.

\section{Conclusiones}

Este estudio nos entrega información local sobre la notificación de EA supuestamente atribuidos a vacunas, permitiendo sentar bases y monitorizar la evolución de éstos a futuro, así como orientar nuevas líneas de investigación, especialmente en vacuna pentavalente. Es importante entonces reforzar la educación sobre la notificación de los ESAVI en Chile, facilitar los medios de notificación e implementar un sistema de recolección de datos que disminuya el riesgo de errores de registro, que contribuya a la monitorización de ESAVI y detectar nuevas señales. Además, es necesario incorporar un sistema de farmacovigilancia activa, a través del cual se logre demostrar o descartar las señales generadas en la vigilancia pasiva a modo de asegurar un estándar más alto de seguridad en vacunas a la población.

Agradecimientos. A Cecilia González Caro y Marcela Avendaño Vigueras pertenecientes al equipo del Departamento de Inmunizaciones del Ministerio de Salud, por su colaboración en la concepción del estudio y la recolección de datos pertenecientes al RNI.

\section{Resumen}

Introducción: Los sistemas de vigilancia pasiva de los eventos supuestamente atribuidos a vacunación o inmu- 
nización (ESAVI) permiten monitorizar efectos adversos (EA) conocidos y pesquisar EA infrecuentes. El objetivo de este estudio fue describir los ESAVI notificados en Chile entre los años 2014 y 2016. Material y Método: Estudio de vigilancia epidemiológica. Se realizó un análisis general y un sub-análisis para vacuna pentavalente. Se calculó la tasa de reporte (TR) global de ESAVI cada 100.000 dosis de vacunas, la TR de ESAVI considerados de interés y el odds ratio de reporte (ROR) de apneas para pentavalente. Resultados: El total de ESAVI notificados fue de 2.552 con una TR global de 9,13. Un 13,4\% de los ESAVI fueron serios. La TR de eventos de interés fue en general menor a las tasas de referencia. La vacuna con mayor TR $(44,3)$ fue pentavalente, con un ROR de apnea versus otras vacunas de 10,5 (IC 95\% 3,77-29,47). Discusión: Este estudio entrega una visión general de los ESAVI notificados en Chile. La TR global fue similar a otras reportadas, pero las de eventos de interés fueron menores a las referencias internacionales. Se muestra una posible asociación entre apneas y vacuna pentavalente.

\section{Referencias bibliográficas}

1.- World Health Organization (WHO), United Nations Children's Fund (UNICEF) WB. State of the world's vaccines and immunization. In 2009 [citado el 11 de marzo de 2018]. Available from: http://apps.who.int/iris/ bitstream/10665/44169/1/9789241563864_eng. pdf.

2.- Ozawa S, Stack M L. Public trust and vaccine acceptance-international perspectives. Hum Vaccines Immunother 2013; 9 (8): 1774-8.

3.- World Health Organization(WHO). Global manual on surveillance of adverse events following immunization. 2014 [citado el 11 de marzo de 2018]; Available from: www.who.int/ about/licensing/copyright_form/en/index.html.

4.- Zhou W, Pool V, Iskander J K, English-Bullard R, Ball R, Wise R P, et al. Surveillance for safety after immunization: Vaccine Adverse Event Reporting System (VAERS)-United States, 1991-2001. MMWR Surveill Summ [Internet]. 2003 [citado el 10 de marzo de 2018]; 52 (1): 1-24. Available from: http:// www.ncbi.nlm.nih.gov/pubmed/12825543.

5.- Shimabukuro T T, Nguyen M, Martin D, Destefano F. Safety monitoring in the Vaccine Adverse Event Reporting System (VAERS) HHS Public Access. Vaccine [Internet]. 2015 [citado el 11 de marzo de 2018]; 33 (36): 4398-405. Available from: https://www.ncbi. nlm.nih.gov/pmc/articles/PMC4632204/pdf/ nihms-732970.pdf.

6.- Group WHOW, Pharmacovigilance V. Definition and Application Pharmacovigilance. Int Ethical Guidel Epidemiol Stud [Internet]. 2009;39-40. Available from: http://www.who. int/vaccine_safety/initiative/tools/CIOMS _ report_WG_vaccine.pdf.

7.- van Puijenbroek E P, Bate A, Leufkens H G M, Lindquist M, Orre R, Egberts A C G. A comparison of measures of disproportionality for signal detection in spontaneous reporting systems for adverse drug reactions.

Pharmacoepidemiol Drug Saf [Internet] 2002; 11 (1): 3-10. Available from: http://www.ncbi. nlm.nih.gov/pubmed/11998548.
8.- Ministerio de Salud. Gobierno de Chile. Guía de Vacunación Segura, sistema de notificación, evaluación e investigación de ESAVI-EPRO [Internet]. 2013. Available from: http://www. ispch.cl/sites/default/files/Guia_de_Vacunacion Segura 2013 0.pdf.

9.- Carbone T, McEntire B, Kissin D, Kelly D, Steinschneider A, Violaris K, et al. Absence of an increase in cardiorespiratory events after Diphtheria-Tetanus-Acellular Pertussis immunization in preterm infants: A randomized, multicenter study. Pediatrics [Internet]. 2008; 121 (5): e1085-90. Available from: http:// pediatrics.aappublications.org/cgi/doi/10.1542/ peds.2007-2059.

10.- Flatz-Jequier A, Posfay-Barbe K M, Pfister R E, Siegrist C A. Recurrence of cardiorespiratory events following repeat DTaP-based combined immunization in very low birth weight premature infants. J Pediatr 2008; 153 (3): 42931.

11.- World Health Organization. Supplementary information on vaccine safety DEPARTMENT OF VACCINES AND BIOLOGICALS The Department of Vaccines and Biologicals thanks the donors whose unspecified financial support has made the production of this document possible. World Health Org Geneva [Internet]. 2000; Available from: http://apps.who.int/iris/ bitstream/10665/66675/1/WHO_V-B_00.36_ eng.pdf.

12.- Kohl K S, Bonhoeffer J, Braun M M, Chen R T, Duclos P, Heijbel H, et al. The Brighton Collaboration: Creating a Global Standard for Case Definitions (and Guidelines) for Adverse Events Following Immunization. [citado el 11 de marzo de 2018]; Available from: https://www.ncbi.nlm.nih.gov/ books/NBK20507/pdf/Bookshelf_NBK20507. pdf.

13.- Rothman K J, Lanes S, Sacks S T. The reporting odds ratio and its advantages over the proportional reporting ratio. Pharmacoepidemiol Drug Saf. 2004;13(8): 519 23.

14.- European Medicines Agency. Screening for adverse reactions in EudraVigilance. 2016 [citadp el 20 de marzo de 2018]; Available from: http://www.ema.europa.eu/docs/ en_GB/document_library/Other/2016/12/ WC500218606.pdf.

15.- Sreelakshmi Sreedhar, Anil Antony N P. Study on the effectiveness and impact of pentavalent vaccination program in India and other south Asian countries. Hum Vaccin Immunother [Internet]. 2014; 10 (7): 2062-5. doi: 10.4161/hv.28785.

16.- Carlsson R M, Claesson B A, Selstam U, Fagerlund E, Granström M, Blondeau C, et al. Safety and immunogenicity of a combined diphtheria-tetanus-acellular pertussis-inactivated polio vaccineHaemophilus influenzae type $\mathrm{b}$ vaccine administered at 2-4-6-13 or 3-5-12 months of age. Pediatr Infect Dis J [Internet]. 1998; 17 (11): 1026-33.PMID: 9849987.

17.- $\mathrm{WHO} \mid \mathrm{WHO}$ vaccine reaction rates information sheets. WHO [Internet]. 2018 [citado el 10 de marzo de 2018]; Available from: http://www.who.int/vaccine_safety/ initiative/tools/vaccinfosheets/en/

18.- Alguacil-Ramos A M, Muelas-Tirado J, Garrigues-Pelufo T M, Portero-Alonso A, Diez-Domingo J, Pastor-Villalba E, et al. Surveillance for adverse events following immunization (AEFI) for 7 years using a computerised vaccination system. Public Health [Internet]. 2016; 135: 66-74. Available from: http://dx.doi.org/10.1016/j. puhe.2015.11.010.

19.- Centers for Diseases Control and Prevention. Paralytic Poliomyelitis-United States, 1980-1994 [Internet]. MMWR: Morb Mortal Wkly Rep 1997; 46: 79-83 [citado el 19 de marzo de 2018]. Available from: https://www.cdc.gov/mmwr/preview/ mmwrhtml/00045949.htm.

20.- Andrus J K, Strebel P M, De Quadros C A, Eive J-MO. Research I Recherche risk of vaccine-associated paralytic poliomyelitis in Latin America, 1989-91 [citado el 19 de marzo de 2018]; Available from: https://www.ncbi. nlm.nih.gov/pmc/articles/PMC2486585/pdf/ bullwho00405-0035.pdf. 
21.- Meinus C, Schmalisch G, Hartenstein S, Proquitté H, Roehr C C. Adverse cardiorespiratory events following primary vaccination of very low birth weight infants. $J$ Pediatr (Rio J) [Internet]. 2012; 88 (2): 137-42. http://dx.doi.org/10.2223/JPED.2182.
22.- Klein N P, Massolo M L, Greene J, Dekker C L, Black S, Escobar G J. Risk Factors for developing apnea after immunization in the Neonatal Intensive Care Unit. Pediatrics [Internet] 2008; 121 (3): 463-9. Available from: http://pediatrics. aappublications.org/cgi/doi/10.1542/peds.20071462.

23.- Who. AEFI Core Variables [Internet]. 2015 [cited 2018 Mar 19]. Available from: http:// www.who.int/vaccine_safety/news/AEFI Core_Variables_2013.pdf?ua $=1$. 